\title{
Umpolung Difunctionalization of Carbonyls via Visible-Light Photoredox Catalytic Radical-Carbanion Relay
}

\author{
Shun Wang, Bei-Yi Cheng, Matea Sršen, and Burkhard König*
}

Cite This: J. Am. Chem. Soc. 2020, 142, 7524-7531

Read Online

ABSTRACT: The combination of photoredox catalysis with the WolffKishner (WK) reaction allows the difunctionalization of carbonyl groups by a radical-carbanion relay sequence (photo-Wolff-Kishner reaction). Photoredox initiated radical addition to $N$-sulfonylhydrazones yields $\alpha$ functionalized carbanions following the WK-type mechanism. With sulfur-centered radicals, the carbanions are further functionalized by reaction with electrophiles including $\mathrm{CO}_{2}$ and aldehydes, whereas $\mathrm{CF}_{3}$ radical addition furnishes a wide range of gem-difluoroalkenes through $\beta$ fluoride elimination of the generated $\alpha-\mathrm{CF}_{3}$ carbanions. More than 80 substrate examples demonstrate the broad applicability of this reaction sequence. A series of investigations including radical inhibition,

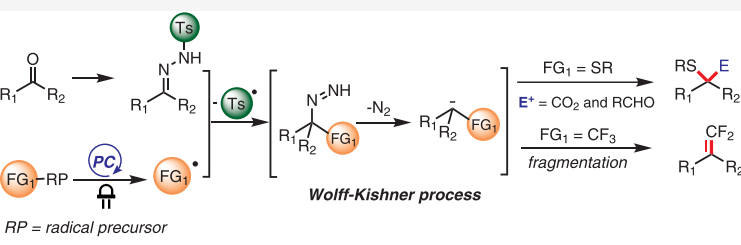

-Combination of photocatalysis with Wolff-Kishner process

- Generation of functionalized alkyl carbanions under mild conditions $->85$ examples \& up to $87 \%$ yield -Mechanistic insights deuterium labeling, fluorescence quenching, cyclic voltammetry, and control experiments support the proposed radical-carbanion relay mechanism.

\section{INTRODUCTION}

The inversion of the inherent polarity of organic functionalities, termed as umpolung, is a key bond-forming strategy in organic synthesis. ${ }^{1}$ The umpolung of a carbonyl group places a negative charge on the carbon atom, making it nucleophilic and prone to attack electrophiles. Carbonyl umpolung is achieved in many ways: Acyl anion equivalents are obtained by the umpolung of electrophilic aldehydes in stoichiometric dithiane chemistry ${ }^{2}$ and catalytic N-heterocyclic carbene (NHC) chemistry. ${ }^{3}$ Synthetically important alkyl carbanion intermediates can be obtained from carbonyl groups using the Wolff-Kishner (WK) reduction. The polarity inversion is accomplished by sequential hydrazone formation, tautomerization, and $\mathrm{N}_{2}$-extrusion to generate a nucleophilic alkyl carbanionic species (Scheme 1A). With elegant modifications from Huang Minlon ${ }^{4}$ and others, ${ }^{5}$ the WK process has evolved over the past century into a powerful carbonyl deoxygenation tool in the synthesis of complex molecules. ${ }^{5}$ Despite being a very effective way of producing carbanions, synthetic applications of this chemistry have long been underexplored considering that the alkyl carbanion in such an umpolung can, in principle, react with many electrophiles other than a proton. Few examples based on the modified WK process have been developed for the construction of $\mathrm{C}-\mathrm{C}$ bonds, wherein highly reactive alkyllithium reagents were employed to react with sulfonylhydrazones. ${ }^{6}$ More recently, pioneering work by Li and co-workers demonstrated the direct functionalization of the carbanion in a Wolff-Kishner reaction by nucleophilic addition to carbonyl compounds, ${ }^{7}$ imines, ${ }^{8} \mathrm{CO}_{2},{ }^{9}$ and Michael acceptors ${ }^{10}$ under ruthenium catalysis. The same group utilized such carbanions in metal-catalyzed Negishi-type coupling, ${ }^{11}$
Heck-type coupling, ${ }^{12}$ Tsuji-Trost alkylation, ${ }^{13}$ and olefination reactions $^{14}$ or metal-free $\mathrm{C}-\mathrm{C}$ bond-forming reactions. ${ }^{15}$ In these cases, the functional groups are installed through metalassisted nucleophilic trapping of nonfunctionalized alkyl carbanions (Scheme 1B). Inspired by the facile generation of carbanions in the classic WK process, we questioned if functionalized carbanions can be produced catalytically for a subsequent nucleophilic reaction allowing the simultaneous installation of two functional groups at a geminal position. The scope of such a reaction sequence has remained unexplored although its realization represents a desirable synthetic tool for carbonyl group functionalization.

As part of our ongoing research activities in photoredox catalytic generation of functionalized carbanions from carbon$\mathrm{yls}^{16}{ }^{16}$ we envisioned that a combination of a conventional WK process with photoredox catalysis might furnish functionalized alkyl carbanions for a subsequent derivatization. In the anticipated radical-carbanion relay sequence, radicals generated by the photoredox catalytic system would be captured by $\mathrm{N}$ sulfonylhydrazone, ${ }^{17}$ thus installing the first functional group. Subsequently, the diazene intermediate, resulting from radical fragmentation, ${ }^{17 a, c, 18}$ enters a similar reaction sequence as involved in the $\mathrm{WK}$ reduction to give functionalized

Received: January 17, 2020

Published: April 1, 2020 
Scheme 1. Umpolung Generation of Alkyl Carbanions from Carbonyls

$$
\begin{aligned}
& \text { (A) Wolff-Kishner reduction }
\end{aligned}
$$

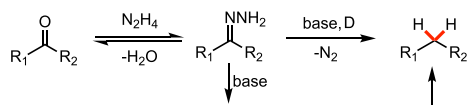

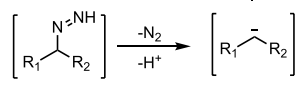

(B) Li's modification

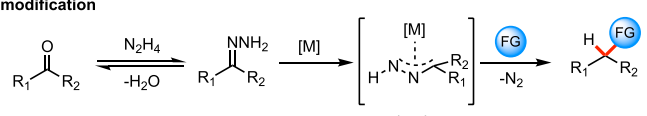

$$
\begin{aligned}
& \begin{array}{l}
\text { carbanion } \\
\text { equivalent }
\end{array}
\end{aligned}
$$

(C) This work: Difunctionalization of carbonyls via photoredox catalytic radical-carbanion relay

a) Reaction design

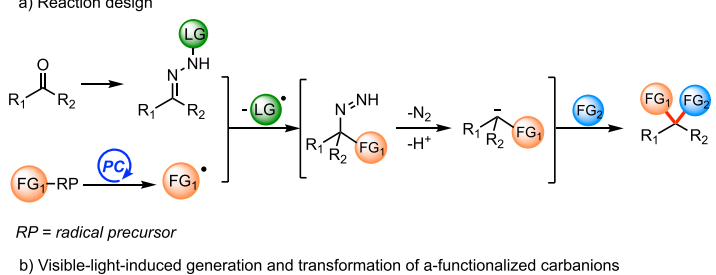

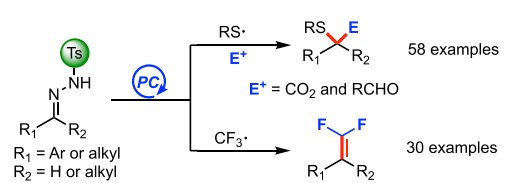

carbanions, which offers a second opportunity for further transformations (Scheme 1C,a). Herein we report the successful implementation of this radical-carbanion relay functionalization concept. The combination of photoredox catalysis with a Wolff-Kishner process allows the facile generation of $\alpha$-sulfenyl and $\alpha-\mathrm{CF}_{3}$ carbanions that undergo further nucleophilic attack or fragmentation, respectively (Scheme 1C,b).

\section{RESULTS AND DISCUSSION}

2.1. Generation of $\alpha$-Sulfenyl Carbanions and Their Reactions with Electrophiles. Carbon-sulfur bonds are found in pharmaceuticals or natural products and are widely used in synthesis. Recent years have witnessed increasing attention to develop an efficient approach to forge $\mathrm{C}-\mathrm{S}$ bonds. ${ }^{19}$ We postulate that photogenerated thiyl radicals ${ }^{20}$ from various thiols can engage in the radical-carbanion relay functionalization sequence. Such a process would yield synthetically useful $\alpha$-sulfenyl carbanions, which are traditionally produced through deprotonation of sulfides with strong bases such as ${ }^{n} \mathrm{BuLi}$ and $\mathrm{NaNH}_{2}{ }^{21}$ Building on the facile carbanion trapping by $\mathrm{CO}_{2}{ }^{22}$ and our continued interest in utilization of $\mathrm{CO}_{2}$ as the $\mathrm{C}_{1}$ feedstock for photocatalytic carboxylation reactions, ${ }^{22 e, 23}$ we selected $N$-tosylhydrazone as the radical acceptor in the anticipated sequence based on the following considerations: (1) they can be easily prepared through condensation of carbonyl compounds with $\mathrm{TsNHNH}_{2}$; (2) after radical addition to $N$-tosylhydrazone, rapid $\beta$-sulfone elimination was anticipated to produce a sulfinyl radical which should undergo single-electron transfer with the photocatalyst. ${ }^{17,24}$ We commenced our study by utilizing aldehyde hydrazone $1 \mathbf{a}$, thiophenol $\mathbf{2 a}$, and $\mathrm{CO}_{2}$ as model substrates for the optimization of the reaction conditions.
After systematic screening of all reaction parameters (see the SI for details), we were delighted to obtain the desired functionalized carboxylic acid $3 \mathrm{a}$ in $81 \%$ yield using $\left[\operatorname{Ir}\left(\mathrm{dFCF}_{3} \text { ppy }\right)_{2} \mathrm{dtbbpy}\right] \mathrm{PF}_{6}(1 \mathrm{~mol} \%)$ under 3 atm of $\mathrm{CO}_{2}$ in DMSO (Table 1 , entry 1 ). Polar solvents like DMSO and

Table 1. Screening of Reaction Conditions for Thiocarboxylation of $\mathrm{N}$-Tosylhydrazone ${ }^{a}$

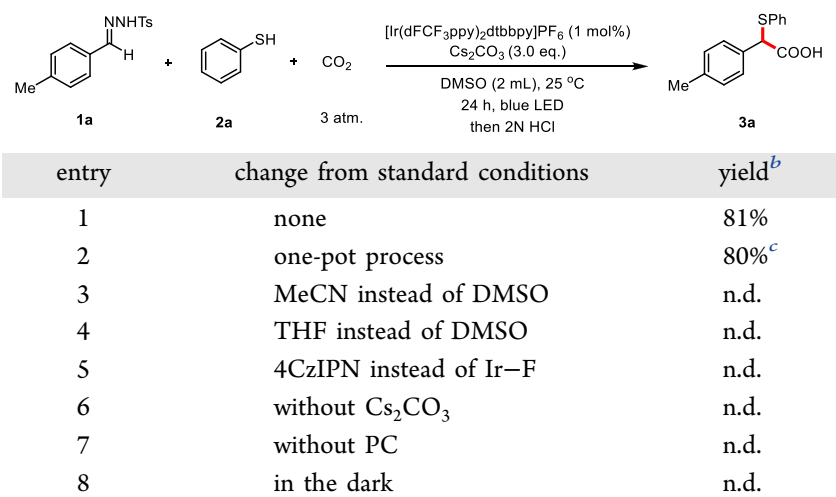

${ }^{a}$ Reaction conditions: compound 1a $(0.2 \mathrm{mmol})$, 2a $(0.3 \mathrm{mmol})$, $\mathrm{Cs}_{2} \mathrm{CO}_{3}(0.6 \mathrm{mmol}),\left[\operatorname{Ir}\left(\mathrm{dFCF}_{3} \mathrm{ppy}\right)_{2} \mathrm{dtbbpy}_{\mathrm{PF}_{6}}(1 \mathrm{~mol} \%)\right.$, and 3 atm of $\mathrm{CO}_{2}$ in $2 \mathrm{~mL}$ of solvent, irradiation with blue LED $(455 \mathrm{~nm})$ at $25{ }^{\circ} \mathrm{C}$ for 24 h. n.d. $=$ not detected. ${ }^{b}$ Yields were determined by ${ }^{1} \mathrm{H}$ NMR analysis of the crude reaction mixture using 1,3,5-trimethoxybenzene as the internal standard. ${ }^{c} \mathbf{1 a}$ was formed in one pot starting from $p$-tolualdehyde and used directly without purification. 4CzIPN = 2,4,5,6-tetra (carbazol-9-yl)isophthalonitrile. $\mathrm{Ir}-\mathrm{F}=[\mathrm{Ir}$ $\left(\mathrm{dFCF}_{3} \text { ppy }\right)_{2} \mathrm{dtbbpy}_{\mathrm{PF}}$. $\mathrm{PC}=$ photocatalyst.

DMF were effective for this thiocarboxylation reaction (see the SI, Table S2). Moreover, we successfully converted $p$ tolualdehyde into the desired product $3 \mathbf{a}$ in one pot by means of a condensation and photocatalytic sequence with similar efficiency (Table 1, entry 2). Rigorous control experiments revealed that photocatalyst, base, and light were crucial for the transformation to occur (Table 1, entries 6-8).

With the optimized reaction conditions in hand, we examined the scope of the method (Table 2). The reaction gave good yields of the corresponding products with a series of aromatic aldehyde-derived $\mathrm{N}$-tosylhydrazones bearing electron-neutral (3a-3c, 3f, $\mathbf{3 g}, \mathbf{3 j}$, and $3 \mathbf{l}$ ), electron-donating (3d, $3 \mathbf{h}$, and $3 \mathbf{o})$, or electron-withdrawing ( $3 \mathbf{i}, 3 \mathbf{k}$, and $3 \mathbf{n})$ groups at para-, meta-, or ortho- positions. The reaction was compatible with $\mathrm{N}$-tosylhydrazones containing two substituents on the aromatic ring, affording the desired carboxylic acids $(3 m-3 o)$ in reasonable yields $(43-58 \%)$. Heterocyclic and naphthalene-containing substituents were also well tolerated by the catalytic system $(3 \mathbf{p}, 3 \mathbf{q})$.

The reaction system could also be extended to $N$ tosylhydrazones derived from ketones, affording a wide range of carboxylic acids with quaternary carbon-centers $(3 \mathbf{r}-3 \mathbf{a e})$. Gratifyingly, functional groups including phenyl (3s), halogen (3t and $3 \mathbf{x}$ ), thiophene (3u), benzofuran (3v), and methoxy (3y) on the aromatic rings of substrates were well tolerated. The reaction proceeded with similar efficiencies for electronrich or electron-poor substrates. Moreover, $N$-tosylhydrazones bearing more sterically hindered substituents at the $\alpha$-position such as ethyl (3aa), isopropyl (3ab), and cyclopropyl (3ac) gave the desired products in good yields, but longer reaction times were required. The reaction could be utilized for the thiocarboxylation of $\mathrm{N}$-tosylhydrazone derived from 4- 
Table 2. Scope of $N$-Tosylhydrazones for Thiocarboxylation $^{a}$

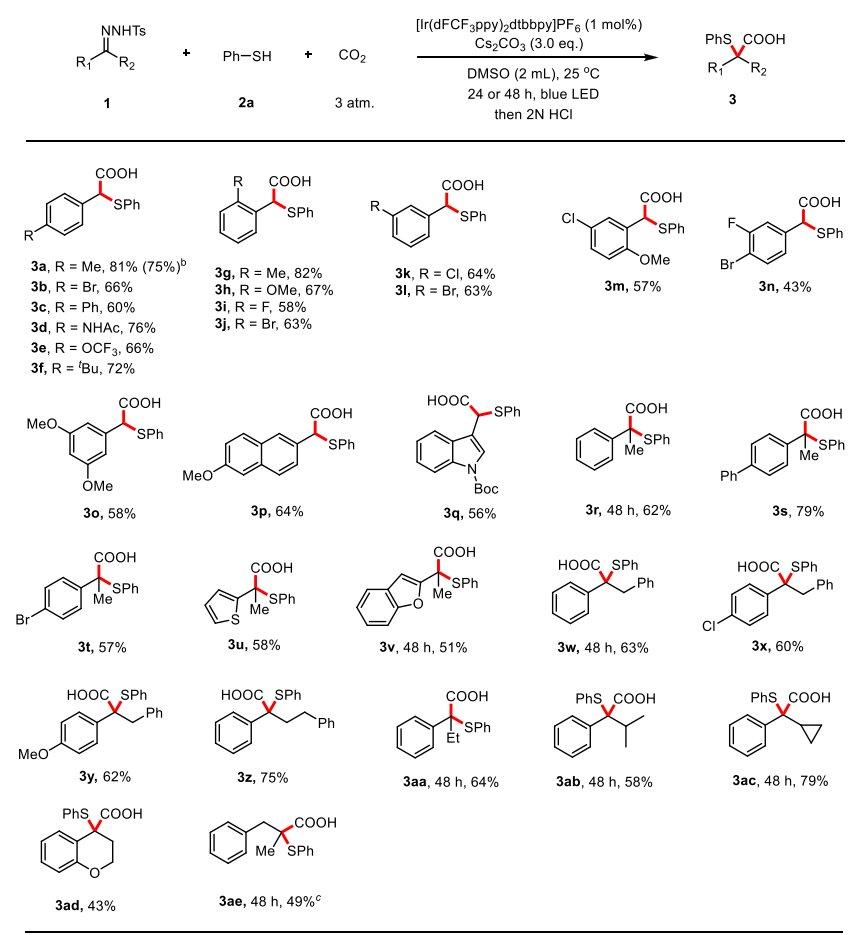

${ }^{a}$ Reaction conditions: unless otherwise noted, all reactions were carried out with $1(0.2 \mathrm{mmol}), 2 \mathrm{a}(0.3 \mathrm{mmol}), \mathrm{Cs}_{2} \mathrm{CO}_{3}(0.6 \mathrm{mmol})$, $\left[\operatorname{Ir}\left(\mathrm{dFCF}_{3} \text { ppy }\right)_{2} \mathrm{dtbbpy}_{\mathrm{PF}_{6}}(1 \mathrm{~mol} \%)\right.$, and 3 atm of $\mathrm{CO}_{2}$ in $2 \mathrm{~mL}$ of DMSO, irradiation with blue LED $(455 \mathrm{~nm})$ at $25{ }^{\circ} \mathrm{C}$ for $24 \mathrm{~h}$, and isolated yields were shown. ${ }^{b} 6 \mathrm{mmol}$ scale, $\mathrm{CO}_{2}$ was bubbled into the reaction continuously. ${ }^{c}$ Reaction was conducted at $0{ }^{\circ} \mathrm{C}$ in DMF (2 $\mathrm{mL}$ )

chromanone, yielding the heterocyclic product 3ad in $43 \%$ yield. To our delight, $N$-tosylhydrazone derived from an aliphatic ketone reacted at $0{ }^{\circ} \mathrm{C}$ yielding product 3 ae in moderate yield. The decreased efficiency and required low reaction temperature were rationalized by the instability of the aliphatic $\alpha$-sulfenly carbanion. Importantly, this reaction is easily scalable, as demonstrated by the gram scale synthesis of $3 \mathbf{a}$ in $75 \%$ yield.

Next, we explored the scope of the reaction with respect to thiols. As shown in Table 3, thiophenols bearing either electron-donating $(\mathbf{4 a - 4 c )}$ or electron-withdrawing groups (4e) on the para position of the aromatic ring reacted smoothly to generate the expected products in mostly good yields. Both ortho- and meta-substituted thiophenols were suitable substrates, affording the products in high yields (71$85 \%)$. However, 4-nitro-thiolphenol failed to give the desired product. Notably, besides aromatic thiophenols, our method could be extended to primary, secondary, and tertiary aliphatic thiols $(4 \mathbf{j}-\mathbf{4 l})$, albeit with moderate efficiencies.

After successful application of this radical-carbanion relay sequence for carboxylation, we tested other electrophiles, like aldehydes or ketones, to realize a visible-light driven Barbiertype reaction. ${ }^{22 b, 25}$ Barbier-type reactions are well-known carbon-carbon forming reactions utilizing the nucleophilic attack of organometallic species to carbonyl compounds. ${ }^{26}$ Using slightly modified reaction conditions, we discovered that photo-Wolff-Kishner generated carbanions can be efficiently trapped with a wide range of aldehydes (Table 4).
Table 3. Scope of the Thiols for Thiocarboxylation ${ }^{a}$

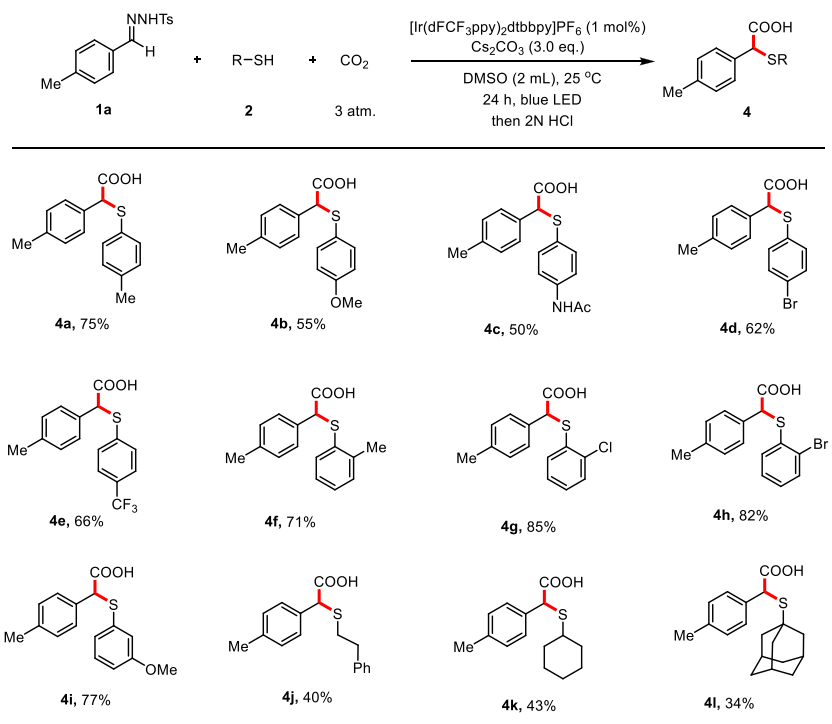

${ }^{a}$ Reaction conditions: unless otherwise noted, all reactions were carried out with $1 \mathrm{a}(0.2 \mathrm{mmol}), 2(0.3 \mathrm{mmol}), \mathrm{Cs}_{2} \mathrm{CO}_{3}(0.6 \mathrm{mmol})$, $\left[\operatorname{Ir}\left(\mathrm{dFCF}_{3} \mathrm{ppy}_{2} \mathrm{dtbbpy}\right] \mathrm{PF}_{6}(1 \mathrm{~mol} \%)\right.$, and $3 \mathrm{~atm}$ of $\mathrm{CO}_{2}$ in $2 \mathrm{~mL}$ of DMSO, irradiation with blue LED $(455 \mathrm{~nm})$ at $25^{\circ} \mathrm{C}$ for $24 \mathrm{~h}$, and isolated yields were shown.

Table 4. Scope of the Aldehydes for Thiohydroxyalkylation ${ }^{a}$

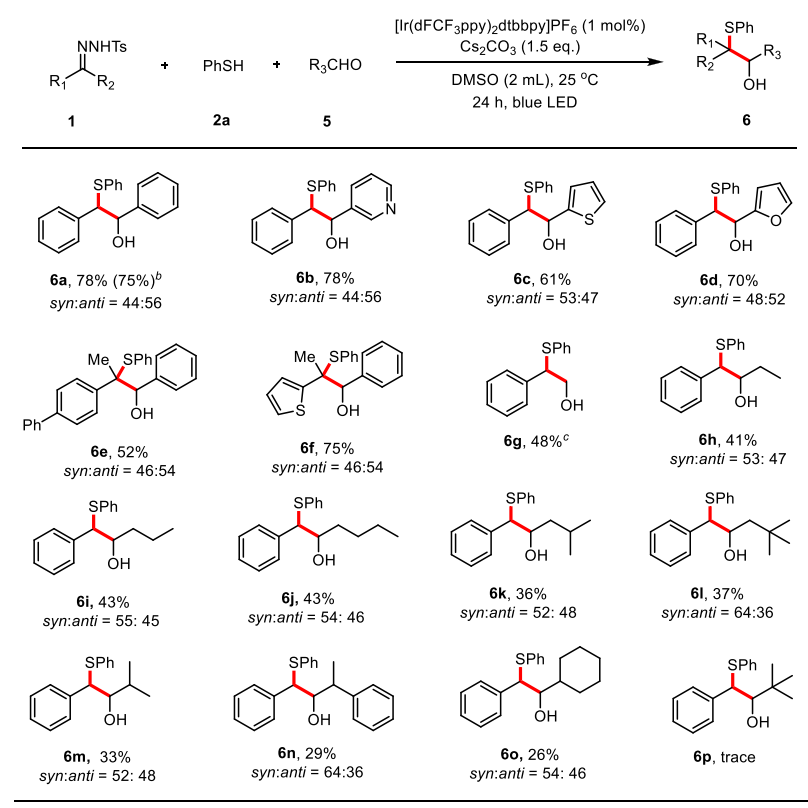

${ }^{a}$ Reaction conditions: unless otherwise noted, all reactions were carried out with $\mathbf{1}(0.2 \mathrm{mmol}), \mathbf{2 a}(0.3 \mathrm{mmol}), \mathbf{5}(0.8 \mathrm{mmol}), \mathrm{Cs}_{2} \mathrm{CO}_{3}$ $(0.3 \mathrm{mmol}),\left[\operatorname{Ir}\left(\mathrm{dFCF}_{3} \mathrm{ppy}_{2} \mathrm{dtbbpy}_{\mathrm{PF}_{6}}(1 \mathrm{~mol} \%)\right.\right.$ in $2 \mathrm{~mL}$ of DMSO, irradiation with blue LED $(455 \mathrm{~nm})$ at $25^{\circ} \mathrm{C}$ for $24 \mathrm{~h}$, and isolated yields were shown. ${ }^{b} 6 \mathrm{mmol}$ scale. ${ }^{c}$ Paraformaldehyde $(0.8$ $\mathrm{mmol})$ and DMSO $(4 \mathrm{~mL})$ were used.

Benzaldehyde reacted smoothly to give the desired alcohol $6 \mathbf{a}$ in $78 \%$ yield. We were delighted to find that heteroaryl aldehydes readily participated in the coupling reaction to give products $\mathbf{6 b}-\mathbf{6} \mathbf{d}$. When ketone-derived $N$-tosylhydrazones were employed, densely functionalized sulfides $(\mathbf{6 e}-\mathbf{6 f})$ were constructed in synthetically useful yields. Besides aromatic 
aldehydes, aliphatic aldehydes bearing short or long chains were suitable electrophiles in our system, giving the desired products in moderate yields $(6 \mathrm{~g}-6 \mathrm{o}))^{27}$ Notably, solid paraformaldehyde reacted to provide the desired product $(6 \mathrm{~g})$ in $48 \%$ yield. This transformation was however sensitive to steric hindrance. The presence of additional substituents at the $\alpha$-carbon on the trapping aldehyde decreased the yield considerably $(6 \mathrm{~m}-60)$, and only a trace amount of the product was detected when pivalaldehyde was employed. Moreover, ketones failed to trap the generated carbanion in the catalytic system, which may be explained by the undesired deprotonation of the $\alpha$-protons to the carbonyl yielding benzyl phenyl sulfide. $^{28}$

2.2. Generation of $\alpha-\mathrm{CF}_{3}$ Carbanions and Their Fragmentation Reactions. Organic molecules containing a fluorine moiety generally exhibit improved reactivity, bioactivity, and metabolic stability compared to their nonfluorinated counterparts. ${ }^{29}$ An important privileged fluorocontaining group is the gem-difluoroethylene moiety based on their unique property in medicinal chemistry. ${ }^{30}$ Moreover, gem-difluoroalkenes are versatile building blocks for the synthesis of other fluorine-containing molecules. ${ }^{31}$ Traditional methods such as Wittig ${ }^{32}$ and Julia ${ }^{33}$ reactions for the synthesis of 1,1-difloroalkenes generally suffer from limited scope, modest efficiency, or harsh conditions. Another efficient pathway is the gem-difluorination of diazo compounds under metal catalytic ${ }^{34}$ or metal-free reaction conditions. ${ }^{35}$ This strategy is generally restricted to aromatic diazo compounds or diazo esters. Recently, several elegant defluorination strategies starting from $\alpha$-trifluoromethyl alkenes based on metal catalysis $^{36}$ or photoredox catalysis ${ }^{37}$ have been developed for the synthesis of gem-difluoroalkenes. Nevertheless, this route requires the presence of trifluoromethyl groups on the alkene moieties, and the product scope is limited by the accessibility of such trifluoromethylated alkenes.

Following the proposal shown in Scheme 1C and encouraged by the success in the generation of $\alpha$-sulfenyl carbanions as described in section 2.1, we wondered whether difluoroalkenes could be produced involving $\mathrm{CF}_{3}$ radicals in the radical-carbanion relay sequence. The feasibility of this approach was supported by the facile E1cB elimination of $\alpha$ $\mathrm{CF}_{3}$ carbanions to yield the difluoroalkenes. ${ }^{37 \mathrm{a}, \mathrm{f}, 38} \mathrm{We}$ used sodium triflinate (Langlois reagent, $\mathrm{CF}_{3} \mathrm{SO}_{2} \mathrm{Na}$ ), a benchstable and commercially available trifluoromethylation reagent, as the $\mathrm{CF}_{3}$ radical precursor and $N$-tosylhydrazone $7 \mathbf{a}$ as the model substrate. ${ }^{39}$ The optimized conditions (see the SI, Tables S5-S7), which include the use of [Ir$\left(\mathrm{dFCF}_{3} \mathrm{ppy}_{2} \mathrm{dtbbpy}_{\mathrm{PF}_{6}}(2 \mathrm{~mol} \%)\right.$ as photocatalyst and $\mathrm{Cs}_{2} \mathrm{CO}_{3}$ (1.5 equiv) as the base in $1 \mathrm{~mL}$ of solvent (DMSO/ acetone $=1 / 1)$, delivered the desired 1,1-difluoroalkene $9 \mathrm{a}$ in $77 \%$ yield (Table 5 , entry 2 ). Likewise, this transformation demonstrated retained efficiency when the reaction was carried out in a one-pot process (Table 5, entry 3). Control experiments indicated that the base, photocatalyst, and light irradiation were essential for this reaction (Table 5, entries 46).

Using the optimized reaction conditions for the gemdifluoroolefination, the scope of this methodology was evaluated. As summarized in Table 6, the reaction proceeded smoothly with a variety of $N$-tosylhydrazones, affording the expected gem-difluoroalkenes in moderate to good yields. The reactions of sodium triflinate with cycloketone-derived $\mathrm{N}$ tosylhydrazones led to the corresponding products $9 \mathbf{a}-9 \mathbf{e}$ in
Table 5. Screening of Reaction Conditions for the 1,1Difluoroolefination of $N$-Tosylhydrazone ${ }^{a}$

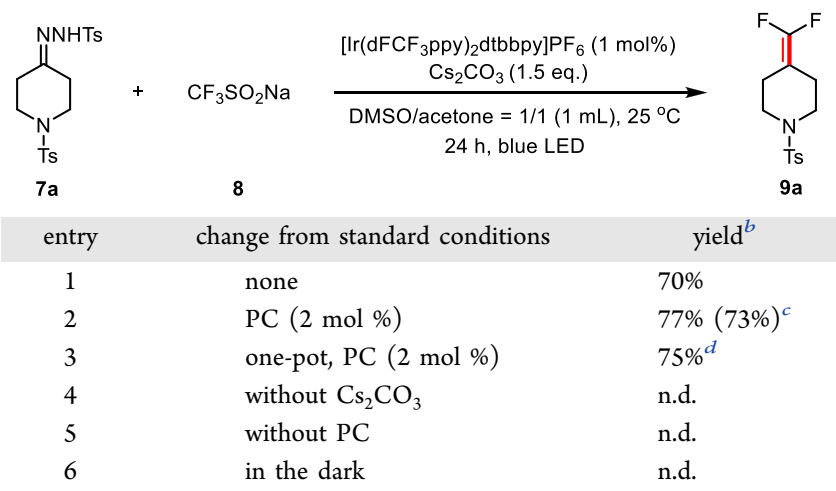

${ }^{a}$ Reaction conditions: compound $7 \mathrm{a}(0.2 \mathrm{mmol}), 8(0.3 \mathrm{mmol})$, $\mathrm{Cs}_{2} \mathrm{CO}_{3}(0.3 \mathrm{mmol}),\left[\operatorname{Ir}\left(\mathrm{dFCF}_{3} \mathrm{ppy}_{2} \mathrm{dtbbpy}_{\mathrm{PF}_{6}}(1 \mathrm{~mol} \%)\right.\right.$ in $1 \mathrm{~mL}$ of solvent, irradiation with blue LED $(455 \mathrm{~nm})$ at $25^{\circ} \mathrm{C}$ for 24 h. n.d. $=$ not detected. ${ }^{b}$ Yields were determined by ${ }^{19} \mathrm{~F}$ NMR analysis of the crude reaction mixture using 4,4'-difluorobenzophenone as the internal standard. ${ }^{c}$ Isolated yield. ${ }^{d} 7 \mathbf{a}$ was formed in one pot starting from corresponding ketone and used directly without purification. PC $=$ photocatalyst.

Table 6. Scope of the gem-Difluoroolefination of $\mathrm{N}$ Tosylhydrazones $^{a}$

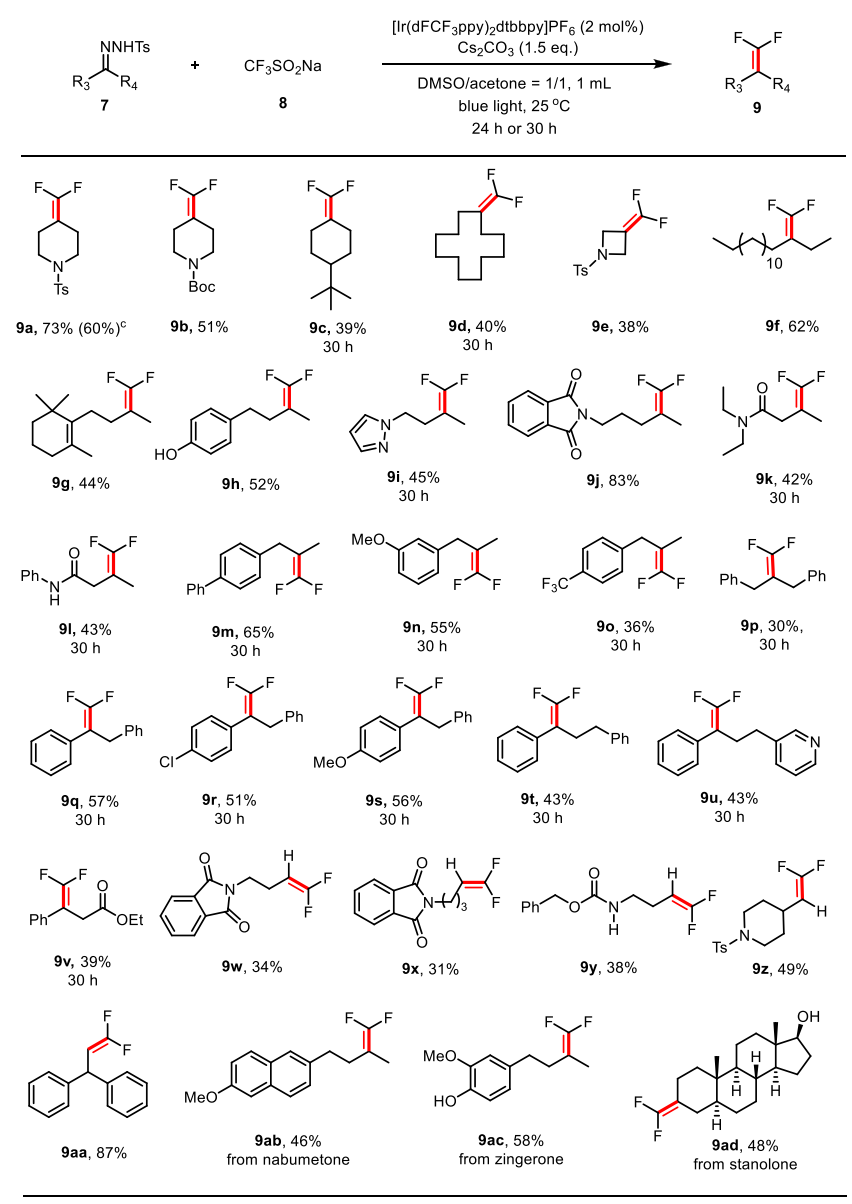

${ }^{a}$ Reaction conditions: unless otherwise noted, all reactions were carried out with $7(0.2 \mathrm{mmol}), 8(0.3 \mathrm{mmol}), \mathrm{Cs}_{2} \mathrm{CO}_{3}(0.3 \mathrm{mmol})$, $\left[\operatorname{Ir}\left(\mathrm{dFCF}_{3} \mathrm{ppy}_{2} \mathrm{dtbbpy}_{\mathrm{PF}_{6}}(2 \mathrm{~mol} \%)\right.\right.$ in $(\mathrm{DMSO} /$ acetone $=1 / 1) 1$ $\mathrm{mL}$, irradiation with blue LED $(455 \mathrm{~nm})$ at $25{ }^{\circ} \mathrm{C}$ for 24 or $30 \mathrm{~h}$, and isolated yields were shown. ${ }^{b} 8 \mathrm{mmol}$ scale, reaction time: $48 \mathrm{~h}$. 
$38-73 \%$ yields. Interestingly, a strained substrate like azetidinone-derivatized $N$-tosylhydrazone could be successfully functionalized yielding difluoroalkene $9 \mathrm{e}$ in modest yield. ${ }^{40}$ Our method could be also extended to acyclic $\mathrm{N}$-tosylhydrazones. For instance, tosylhydrazone derived from 3hexadecanone performed well in our reaction affording the desired product $9 f$ in $62 \%$ yield. Moreover, the mild reaction conditions were compatible with ketone-based tosylhydrazones bearing a wide range of functional groups including alkene (9g), phenol (9h), and amide (9j-9l). With $N$-tosylhydrazones derived from phenylacetones, functional groups such as phenyl, methoxy, and trifluoromethyl on the aromatic ring were well tolerated $(9 \mathbf{9}-\mathbf{9 o})$. A sterically hindered substrate $7 \mathbf{p}$ participated in the reaction well to yield the gemdifluoroalkene. Tosylhydrazones derived from aromatic ketones were also applicable affording the desired products $(\mathbf{9 q}-\mathbf{9 u})$ in reasonable yields. The reactions proceeded smoothly with heterocycle-containing substrates (e.g., pyrazole $7 \mathbf{i}$ and pyridine $7 \mathbf{u}$ ). Notably, ester groups on the carbon chain remained untouched $(9 \mathbf{v})$. This catalytic system was also suitable for aliphatic aldehyde-based $\mathrm{N}$-tosylhydrazones, delivering the corresponding products in moderate to excellent yields $(9 \mathbf{w}-9 \mathbf{a a})$. The utility of this method was further demonstrated by applying it to functionalize structurally and functionally complex natural products like nabumetone, zingerone, and stanolone, providing the desired products $(9 \mathbf{a b}-9 \mathrm{ad})$ in good yields.

2.3. Reaction Mechanism. To gain insights into the reaction mechanism, a series of spectroscopic investigations and control experiments were conducted. First, no desired products were detected when the radical scavenger TEMPO (2.5 equiv) was added to the thiocarboxylation or gemdifluoroolefination reaction. The radical nature of this type of reaction was further confirmed by the formation of TEMPO$\mathrm{SPh}$ and TEMPO- $\mathrm{CF}_{3}$ adducts, which were detected by the HRMS (Scheme 2A and the SI). Based on our results and literature reports about the radical functionalization of $\mathrm{N}$ sulfonylhydrazones, ${ }^{17}$ we postulate that the sulfur-centered radical and $\mathrm{CF}_{3}$ radical follow a similar mechanism to react with $\mathrm{N}$-tosylhydrazones to give the carbanions. We chose the thiocarboxylation reaction as a model reaction to study the mechanism more closely.

A control experiment using sodium thiophenolate in place of the corresponding thiophenol 2a yielded the desired carboxylic acid 3a in $73 \%$ yield. Moreover, we found that the product could be generated in $50 \%$ yield with sodium thiophenolate even in the absence of $\mathrm{Cs}_{2} \mathrm{CO}_{3}$, suggesting that the base $\left(\mathrm{Cs}_{2} \mathrm{CO}_{3}\right)$ merely serves to deprotonate the thiols (Scheme 2B). The Stern-Volmer luminescence quenching experiments revealed that sodium thiophenolate quenches the excited state of the photocatalyst much more efficiently than $\mathrm{N}$-tosylhydrazone 1a and thiophenol 2a (see the SI, Figures S8-S11). Light "on-off" experiments indicated that continuous light irradiation was essential for the reaction to proceed (see the SI). Additionally, the quantum yield of this transformation was determined to be $2.1 \%$. Hence, a radical chain process is unlikely for this reaction. The combined results suggest a transient sulfur-centered radical, generated by single-electron oxidation of thiophenolate by the excited state of photocatalyst in a reductive quenching photocatalytic cycle.

Further control experiments showed that (4-methylbenzyl)(phenyl)sulfide could be obtained in 69\% yield in the absence of $\mathrm{CO}_{2}$ (Scheme 2C). This finding suggests that the sulfur-
Scheme 2. Mechanistic Studies
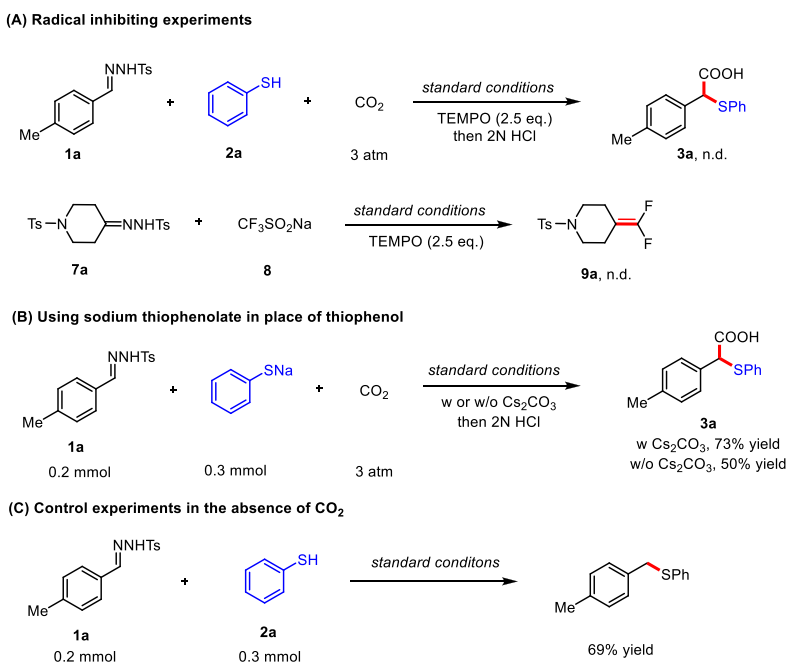

(D) Deuterium incorporation experiments

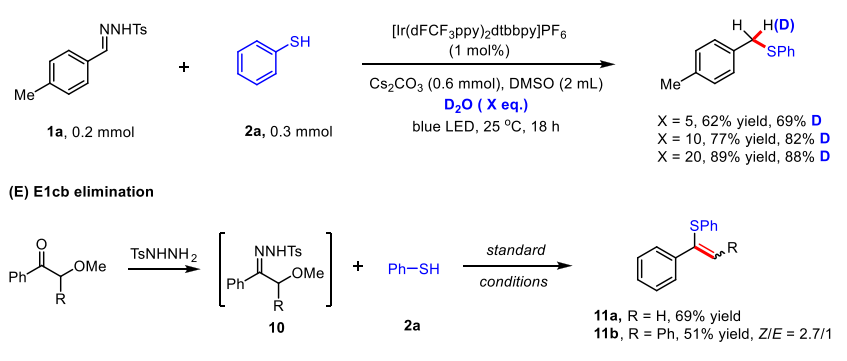

centered radical could interact with $N$-tosylhydrazone 1a irrespective of the existence of $\mathrm{CO}_{2}$. The result is in accordance with the hypothesis that a transient $\alpha$-sulfenyl carbanion might occur in the reaction process. On this basis, we conducted isotope-labeling experiments. Indeed, when $\mathrm{D}_{2} \mathrm{O}$ was added in the absence of $\mathrm{CO}_{2}$, up to $88 \%$ deuterium incorporation into sulfide was observed (Scheme 2D). In addition, a carbanion intermediate should in principle undergo E1cB elimination when the adjacent carbon atom bears an appropriate leaving group. ${ }^{41}$ Therefore, $N$-tosylhydrazones bearing a methoxyl group at the vicinal carbon were prepared and subjected to the standard reaction conditions in the absence of $\mathrm{CO}_{2}$ giving the corresponding alkenes $11 \mathbf{a}$ and $\mathbf{1 1} \mathbf{b}$ in good yields (Scheme $2 \mathrm{E}$ ).

Based on the above experimental evidence and mechanistic pathways reported in the literature, we propose a plausible mechanism as depicted in Scheme 3 for the reported photocatalytic generation of functionalized carbanions. Initially, the photoexcited state of $\left[\operatorname{II}^{\mathrm{III}}\left(\mathrm{dFCF}_{3} \text { ppy }\right)_{2} \mathrm{dtbbpy}\right]^{+}$ $\left(E_{1 / 2}\left[{ }^{*} \mathrm{Ir}^{\mathrm{III}}{ }^{\mathrm{II}}\right]=+1.21 \mathrm{~V} \text { vs } \mathrm{SCE}\right)^{42}$ is reductively quenched by sodium triflinate $\left(E_{\mathrm{ox}}=+1.05 \mathrm{~V} \text { vs } \mathrm{SCE}\right)^{22 \mathrm{a}}$ or thiophenolate $\left(E_{\mathrm{ox}}=\sim 0.75 \mathrm{~V}\right.$ vs SCE $),{ }^{43}$ formed through the deprotonation of thiophenol by base, affording a sulfurcentered radical and a $\mathrm{CF}_{3}$ radical, respectively. Subsequent radical addition to the $\mathrm{C}=\mathrm{N}$ bond of $N$-tosylhydrazone generates the aminyl radical species $\mathrm{A}^{44}$ Fragmentation of the arenesulfonyl radical from intermediate $\mathbf{A}$ leads to a functionalized diazene intermediate $\mathbf{B},^{18}$ and the following WolffKishner type $\mathrm{N}_{2}$ extrusion process proceeds to give $\alpha-\mathrm{CF}_{3}$ or sulfur carbanion $\mathrm{C}$ for further reactions. In the case of the $\alpha$ sulfenyl carbanion, subsequent nucleophilic attack to $\mathrm{CO}_{2}$ or aliphatic aldehydes give carboxylic acids or alcohols. When $\alpha$ $\mathrm{CF}_{3}$ carbanions were produced, $\beta$-fluoride elimination 
Scheme 3. Proposed Mechanism of the Photo-WolffKishner Carbanion Generation

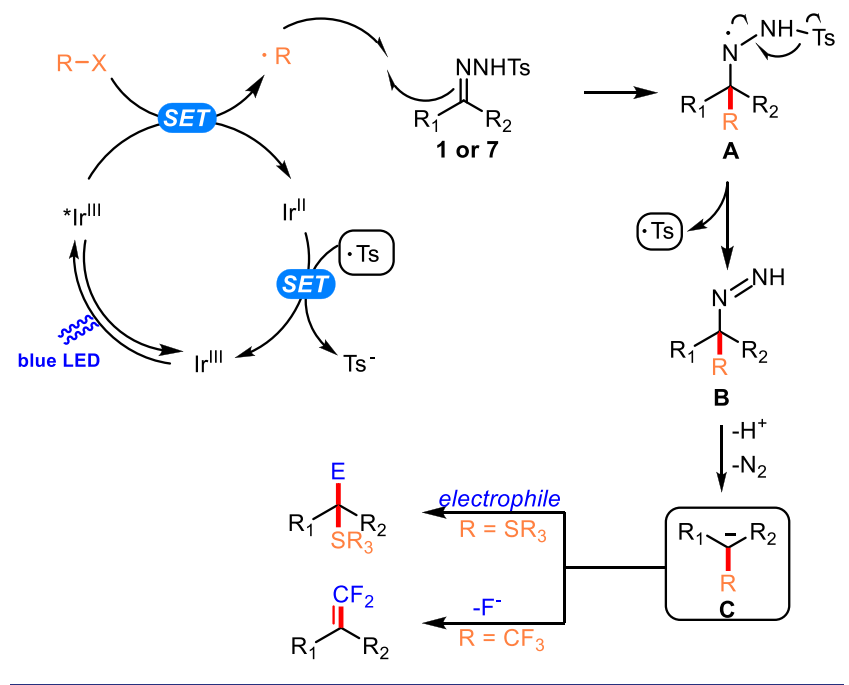

occurred to furnish the gem-difluoroalkenes. Finally, singleelectron transfer (SET) from the reduced photoredox catalyst $\operatorname{Ir}^{\mathrm{II}}\left(E_{1 / 2}\left[\mathrm{Ir}_{\mathrm{III}} / \mathrm{II}\right]=-1.37 \mathrm{~V} \text { vs } \mathrm{SCE}\right)^{42}$ to the arenesulfonyl radical $\left(E_{\text {red }}=+0.50 \mathrm{~V} \text { vs SCE }\right)^{24 \mathrm{~b}}$ yields a sulfinate anion and regenerates the photocatalyst.

\section{CONCLUSION}

In summary, we have established a new reaction sequence for the generation of $\alpha$-functionalized alkyl carbanions through the merger of photoredox catalytic radical generation with the classic Wolff-Kishner (WK) reaction. This radical-carbanion relay for carbonyl functionalization involves the radical addition to $\mathrm{N}$-sulfonylhydrazones, which enables the formation of $\alpha$-substituted carbanion intermediates. Subsequent reaction with electrophiles including $\mathrm{CO}_{2}$ and aldehydes or fragmentation results in thiocarboxylation, thiohydroxyalkylation, and gem-difluoroolefination with broad substrate scope and good tolerance of many functional groups. Mechanistic studies support the hypothesis that a tandem photocatalytic radical addition/Wolff-Kishner process starting from $\mathrm{N}$-sulfonylhydrazones facilitates the formation of the carbanion. This strategy greatly expands the synthetic potential of WolffKishner reaction. Further studies aiming to generate nonstabilized carbanions by this strategy are currently under investigation.

\section{ASSOCIATED CONTENT}

\section{(5) Supporting Information}

The Supporting Information is available free of charge at https://pubs.acs.org/doi/10.1021/jacs.0c00629.

Experiments and spectral details for all new compounds and all reactions reported (PDF)

\section{AUTHOR INFORMATION}

\section{Corresponding Author}

Burkhard König - Institute of Organic Chemistry, Faculty of Chemistry and Pharmacy, University of Regensburg, D-93040 Regensburg, Germany; (1) orcid.org/0000-0002-6131-4850; Email: burkhard.koenig@ur.de

\section{Authors}

Shun Wang - Institute of Organic Chemistry, Faculty of Chemistry and Pharmacy, University of Regensburg, D-93040 Regensburg, Germany

Bei-Yi Cheng - Institute of Organic Chemistry, Faculty of Chemistry and Pharmacy, University of Regensburg, D-93040 Regensburg, Germany

Matea Sršen - Institute of Organic Chemistry, Faculty of Chemistry and Pharmacy, University of Regensburg, D-93040 Regensburg, Germany

Complete contact information is available at:

https://pubs.acs.org/10.1021/jacs.0c00629

\section{Notes}

The authors declare no competing financial interest.

\section{ACKNOWLEDGMENTS}

This work was supported by the German Science Foundation (DFG) (KO 1537/18-1). This project has received funding from the European Research Council (ERC) under the European Unions Horizon 2020 research and innovation programme (grant agreement 741623). S.W. (CSC student number 201606280052) and B.-Y.C. thank the China Scholarship Council (CSC) for a predoctoral fellowship. We thank Dr. Rudolf Vasold (University of Regensburg) for his assistance in GC-MS measurements and Ms. Regina Hoheisel (University of Regensburg) for her assistance in cyclic voltammetry measurements.

\section{REFERENCES}

(1) Seebach, D. Methods of Reactivity Umpolung. Angew. Chem., Int. Ed. Engl. 1979, 18, 239-258.

(2) (a) Gröbel, B.-T.; Seebach, D. Umpolung of the Reactivity of Carbonyl Compounds through Sulfur-Containing Reagents. Synthesis 1977, 1977, 357-402. (b) Seebach, D.; Corey, E. J. Generation and Synthetic Applications of 2-lithio-1,3-dithianes. J. Org. Chem. 1975, 40, 231-237. (c) Smith, A. B.; Adams, C. M. Evolution of DithianeBased Strategies for the Construction of Architecturally Complex Natural Products. Acc. Chem. Res. 2004, 37, 365-377.

(3) (a) Bugaut, X.; Glorius, F. Organocatalytic Umpolung: Nheterocyclic Carbenes and Beyond. Chem. Soc. Rev. 2012, 41, 35113522. (b) Flanigan, D. M.; Romanov-Michailidis, F.; White, N. A.; Rovis, T. Organocatalytic Reactions Enabled by N-Heterocyclic Carbenes. Chem. Rev. 2015, 115, 9307-9387. (c) Marion, N.; DíezGonzález, S.; Nolan, S. P. N-Heterocyclic Carbenes as Organocatalysts. Angew. Chem., Int. Ed. 2007, 46, 2988-3000.

(4) Huang-Minlon. A Simple Modification of the Wolff-Kishner Reduction. J. Am. Chem. Soc. 1946, 68, 2487-2488.

(5) Lewis, D. E. The Wolff-Kishner Reduction and Related Reactions: Discovery and Development; Elsevier, 2019.

(6) (a) Vedejs, E.; Stolle, W. T. Reductive alkylation of aldehyde tosylhydrazones with organolithium reagents. Tetrahedron Lett. 1977, 18, 135-138. (b) Vedejs, E.; Dolphin, J.; Stolle, W. A new olefin synthesis: condensation of aldehyde tosylhydrazones with stabilized carbanions. J. Am. Chem. Soc. 1979, 101, 249-251. (c) Myers, A. G.; Kukkola, P. J. Stereoselective synthesis of olefins from silylated sulfonylhydrazones. J. Am. Chem. Soc. 1990, 112, 8208-8210. (d) Myers, A. G.; Movassaghi, M. Highly Efficient Methodology for the Reductive Coupling of Aldehyde Tosylhydrazones with Alkyllithium Reagents. J. Am. Chem. Soc. 1998, 120, 8891-8892.

(7) Wang, H.; Dai, X.-J.; Li, C.-J. Aldehydes as Alkyl Carbanion Equivalents for Additions to Carbonyl Compounds. Nat. Chem. 2017, 9, 374-378.

(8) Chen, N.; Dai, X.-J.; Wang, H.; Li, C.-J. Umpolung Addition of Aldehydes to Aryl Imines. Angew. Chem., Int. Ed. 2017, 56, 62606263. 
(9) Yan, S.-S.; Zhu, L.; Ye, J.-H.; Zhang, Z.; Huang, H.; Zeng, H.; Li, C.-J.; Lan, Y.; Yu, D.-G. Ruthenium-Catalyzed Umpolung Carboxylation of Hydrazones with $\mathrm{CO}_{2}$. Chem. Sci. 2018, 9, 4873-4878.

(10) Dai, X.-J.; Wang, H.; Li, C.-J. Carbonyls as Latent Alkyl Carbanions for Conjugate Additions. Angew. Chem., Int. Ed. 2017, 56, 6302-6306.

(11) Tang, J.; Lv, L.; Dai, X.-J.; Li, C.-C.; Li, L.; Li, C.-J. NickelCatalyzed Cross-Coupling of Aldehydes with Aryl Halides via Hydrazone Intermediates. Chem. Commun. 2018, 54, 1750-1753.

(12) Lv, L.; Zhu, D.; Li, C.-J. Direct Dehydrogenative Alkyl HeckCouplings of Vinylarenes with Umpolung Aldehydes Catalyzed by Nickel. Nat. Commun. 2019, 10, 715.

(13) Zhu, D.; Lv, L.; Li, C.-C.; Ung, S.; Gao, J.; Li, C.-J. Umpolung of Carbonyl Groups as Alkyl Organometallic Reagent Surrogates for Palladium-Catalyzed Allylic Alkylation. Angew. Chem., Int. Ed. 2018, 57, 16520-16524.

(14) Wei, W.; Dai, X.-J.; Wang, H.; Li, C.; Yang, X.; Li, C.-J. Ruthenium(II)-Catalyzed Olefination via Carbonyl Reductive CrossCoupling. Chem. Sci. 2017, 8, 8193-8197.

(15) Zeng, H.; Luo, Z.; Han, X.; Li, C.-J. Metal-Free Construction of the $\mathrm{C}\left(\mathrm{sp}^{3}\right)-\mathrm{CF}_{3}$ Bond: Trifluoromethylation of Hydrazones with Togni's Reagent under Mild Conditions. Org. Lett. 2019, 21, 59485951.

(16) Wang, S.; Lokesh, N.; Hioe, J.; Gschwind, R. M.; König, B. Photoinitiated Carbonyl-Metathesis: Deoxygenative Reductive Olefination of Aromatic Aldehydes via Photoredox Catalysis. Chem. Sci. 2019, 10, 4580-4587.

(17) (a) Kim, S.; Cho, J. R. Radical Cyclization of Mesitylsulfonylhydrazones. Synlett 1992, 1992, 629-630. (b) Dao, H. T.; Li, C.; Michaudel, Q.; Maxwell, B. D.; Baran, P. S. Hydromethylation of Unactivated Olefins. J. Am. Chem. Soc. 2015, 137, 8046-8049. (c) Campbell, N. E.; Sammis, G. M. Single-Electron/Pericyclic Cascade for the Synthesis of Dienes. Angew. Chem., Int. Ed. 2014, 53, 6228-6231.

(18) (a) Baldwin, J. E.; Bottaro, J. C.; Kolhe, J. N.; Adlington, R. M. Azo Anions in Synthesis. Use of Trityl- and Diphenyl-4Pyridylmethyl-Hydrazones for Reductive C-C Bond Formation from Aldehydes and Ketones. J. Chem. Soc., Chem. Commun. 1984, 22-23. (b) Baldwin, J. E.; Adlington, R. M.; Bottaro, J. C.; Kolhe, J. N.; Newington, I. M.; Perry, M. W. D. Azo Anions in Synthesis: Use of Trityl- and Diphenyl-4-pyridylmethylhydrazones for Reductive C-C Bond Formation. Tetrahedron 1986, 42, 4235-4246. (c) Reyes, J. R.; Rawal, V. H. Reductive Chlorination and Bromination of Ketones via Trityl Hydrazones. Angew. Chem. 2016, 128, 3129-3132.

(19) (a) Chauhan, P.; Mahajan, S.; Enders, D. Organocatalytic Carbon-Sulfur Bond-Forming Reactions. Chem. Rev. 2014, 114, 8807-8864. (b) Shen, C.; Zhang, P.; Sun, Q.; Bai, S.; Hor, T. S. A.; Liu, X. Recent Advances in C-S Bond Formation via C-H Bond Functionalization and Decarboxylation. Chem. Soc. Rev. 2015, 44, 291-314. (c) Feng, M.; Tang, B.; Liang, S. H.; Jiang, X. Sulfur Containing Scaffolds in Drugs: Synthesis and Application in Medicinal Chemistry. Curr. Top. Med. Chem. 2016, 16, 1200-1216. (d) Wang, N.; Saidhareddy, P.; Jiang, X. Construction of SulfurContaining Moieties in the Total Synthesis of Natural Products. Nat. Prod. Rep. 2020, 37, 246-275.

(20) (a) Guo, W.; Tao, K.; Tan, W.; Zhao, M.; Zheng, L.; Fan, X. Recent Advances in Photocatalytic C-S/P-S Bond Formation via the Generation of Sulfur Centered Radicals and Functionalization. Org. Chem. Front. 2019, 6, 2048-2066. (b) Wimmer, A.; König, B. Photocatalytic Formation of Carbon-Sulfur Bonds. Beilstein J. Org. Chem. 2018, 14, 54-83.

(21) Fernández de la Pradilla, R.; Viso, A. Alkylation of $\alpha$-SulfurContaining Carbanions. Comprehensive Organic Synthesis: Second Edition 2014, 3, 157-208.

(22) (a) Yatham, V. R.; Shen, Y.; Martin, R. Catalytic Intermolecular Dicarbofunctionalization of Styrenes with $\mathrm{CO}_{2}$ and Radical Precursors. Angew. Chem., Int. Ed. 2017, 56, 10915-10919. (b) Liao, L.-L.; Cao, G.-M.; Ye, J.-H.; Sun, G.-Q.; Zhou, W.-J.; Gui, Y.-Y.; Yan, S.-S.; Shen, G.; Yu, D.-G. Visible-Light-Driven External-
Reductant-Free Cross-Electrophile Couplings of Tetraalkyl Ammonium Salts. J. Am. Chem. Soc. 2018, 140, 17338-17342. (c) Hou, J.; Ee, A.; Cao, H.; Ong, H.-W.; Xu, J.-H.; Wu, J. Visible-Light-Mediated Metal-Free Difunctionalization of Alkenes with $\mathrm{CO}_{2}$ and Silanes or $\mathrm{C}\left(\mathrm{sp}^{3}\right)-\mathrm{H}$ Alkanes. Angew. Chem., Int. Ed. 2018, 57, 17220-17224. (d) Yoo, W.-J.; Kondo, J.; Rodríguez-Santamaría, J. A.; Nguyen, T. V. Q.; Kobayashi, S. Efficient Synthesis of $\alpha$-Trifluoromethyl Carboxylic Acids and Esters through Fluorocarboxylation of gem-Difluoroalkenes. Angew. Chem., Int. Ed. 2019, 58, 6772-6775. (e) Meng, Q.-Y.; Schirmer, T. E.; Berger, A. L.; Donabauer, K.; König, B. Photocarboxylation of Benzylic C-H Bonds. J. Am. Chem. Soc. 2019, 141, 11393-11397.

(23) (a) Meng, Q.-Y.; Wang, S.; König, B. Carboxylation of Aromatic and Aliphatic Bromides and Triflates with $\mathrm{CO}_{2}$ by Dual Visible-Light-Nickel Catalysis. Angew. Chem., Int. Ed. 2017, 56, 13426-13430. (b) Meng, Q.-Y.; Wang, S.; Huff, G. S.; König, B. Ligand-Controlled Regioselective Hydrocarboxylation of Styrenes with $\mathrm{CO}_{2}$ by Combining Visible Light and Nickel Catalysis. J. Am. Chem. Soc. 2018, 140, 3198-3201. (c) Sahoo, B.; Bellotti, P.; JuliáHernández, F.; Meng, Q.-Y.; Crespi, S.; König, B.; Martin, R. SiteSelective, Remote $\mathrm{sp}^{3} \mathrm{C}-\mathrm{H}$ Carboxylation Enabled by the Merger of Photoredox and Nickel Catalysis. Chem. - Eur. J. 2019, 25, 90019005.

(24) (a) Noble, A.; MacMillan, D. W. C. Photoredox $\alpha$-Vinylation of $\alpha$-Amino Acids and N-Aryl Amines. J. Am. Chem. Soc. 2014, 136, 11602-11605. (b) Heitz, D. R.; Rizwan, K.; Molander, G. A. VisibleLight-Mediated Alkenylation, Allylation, and Cyanation of Potassium Alkyltrifluoroborates with Organic Photoredox Catalysts. J. Org. Chem. 2016, 81, 7308-7313.

(25) Berger, A. L.; Donabauer, K.; König, B. Photocatalytic Barbier Reaction - Visible-Light Induced Allylation and Benzylation of Aldehydes and Ketones. Chem. Sci. 2018, 9, 7230-7235.

(26) (a) Barbier, P. Synthesis of dimethylheptenol. C. R. Acad. Sci. 1899, 128, 110-111. (b) Yamamoto, Y.; Asao, N. Selective Reactions Using Allylic Metals. Chem. Rev. 1993, 93, 2207-2293.

(27) In the examples of $\mathbf{6 h}$ and $\mathbf{6 i}$, benzyl phenyl sulfide was formed in $29 \%$ and $32 \%$ yield, respectively.

(28) Nakamura, S.; Nakagawa, R.; Watanabe, Y.; Toru, T. Highly Enantioselective Reactions of Configurationally Labile $\alpha$-Thioorganolithiums Using Chiral Bis(oxazoline)s via Two Different Enantiodetermining Steps. J. Am. Chem. Soc. 2000, 122, 1134011347.

(29) (a) Müller, K.; Faeh, C.; Diederich, F. Fluorine in Pharmaceuticals: Looking Beyond Intuition. Science 2007, 317, 1881-1886. (b) Hagmann, W. K. The Many Roles for Fluorine in Medicinal Chemistry. J. Med. Chem. 2008, 51, 4359-4369. (c) Gillis, E. P.; Eastman, K. J.; Hill, M. D.; Donnelly, D. J.; Meanwell, N. A. Applications of Fluorine in Medicinal Chemistry. J. Med. Chem. 2015, $58,8315-8359$.

(30) (a) Gouverneur, V., Müller, K., Eds. Fluorine in Pharmaceutical and Medicinal Chemistry: From Biophysical Aspects to Clinical Applications; Imperial College Press: London, 2012. (b) Ojima, I., Ed. Fluorine in Medicinal Chemistry and Chemical Biology; WileyBlackwell: West Sussex, UK, 2009.

(31) (a) Chelucci, G. Synthesis and Metal-Catalyzed Reactions of gem-Dihalovinyl Systems. Chem. Rev. 2012, 112, 1344-1462. (b) Zhang, X.; Cao, S. Recent advances in the synthesis and C-F functionalization of gem-difluoroalkenes. Tetrahedron Lett. 2017, 58, 375-392. (c) Koley, S.; Altman, R. A. Recent Advances in Transition Metal-catalyzed Functionalization of gem-Difluoroalkenes. Isr. J. Chem. 2020, in press. DOI: $10.1002 /$ ijch.201900173.

(32) (a) Zheng, J.; Cai, J.; Lin, J.-H.; Guo, Y.; Xiao, J.-C. Synthesis and Decarboxylative Wittig Reaction of Difluoromethylene Phosphobetaine. Chem. Commun. 2013, 49, 7513-7515. (b) Burton, D. J.; Yang, Z.-Y.; Qiu, W. Fluorinated Ylides and Related Compounds. Chem. Rev. 1996, 96, 1641-1716.

(33) (a) Zhao, Y.; Huang, W.; Zhu, L.; Hu, J. Difluoromethyl 2Pyridyl Sulfone: A New gem-Difluoroolefination Reagent for Aldehydes and Ketones. Org. Lett. 2010, 12, 1444-1447. (b) Gao, 
B.; Zhao, Y.; Hu, M.; Ni, C.; Hu, J. gem-Difluoroolefination of Diaryl Ketones and Enolizable Aldehydes with Difluoromethyl 2-Pyridyl Sulfone: New Insights into the Julia-Kocienski Reaction. Chem. - Eur. J. 2014, 20, 7803-7810.

(34) (a) Zhang, Z.; Zhou, Q.; Yu, W.; Li, T.; Wu, G.; Zhang, Y.; Wang, J. Cu(I)-Catalyzed Cross-Coupling of Terminal Alkynes with Trifluoromethyl Ketone N-Tosylhydrazones: Access to 1,1-Difluoro1,3-enynes. Org. Lett. 2015, 17, 2474-2477. (b) Hu, M.; He, Z.; Gao, B.; Li, L.; Ni, C.; Hu, J. Copper-Catalyzed gem-Difluoroolefination of Diazo Compounds with $\mathrm{TMSCF}_{3}$ via C-F Bond Cleavage. J. Am. Chem. Soc. 2013, 135, 17302-17305. (c) Yang, Z.; Möller, M.; Koenigs, R. M. Synthesis of gem-Difluoro Olefins through C-H Functionalization and $\beta$-Fluoride Elimination Reactions. Angew. Chem., Int. Ed. 2020, 59, 5572-5576.

(35) Hu, M.; Ni, C.; Li, L.; Han, Y.; Hu, J. gem-Difluoroolefination of Diazo Compounds with $\mathrm{TMSCF}_{3}$ or $\mathrm{TMSCF}_{2} \mathrm{Br}$ : TransitionMetal-Free Cross-Coupling of Two Carbene Precursors. J. Am. Chem. Soc. 2015, 137, 14496-14501.

(36) (a) Ding, D.; Lan, Y.; Lin, Z.; Wang, C. Synthesis of gemDifluoroalkenes by Merging Ni-Catalyzed C-F and C-C Bond Activation in Cross-Electrophile Coupling. Org. Lett. 2019, 21, 2723-2730. (b) Lan, Y.; Yang, F.; Wang, C. Synthesis of gemDifluoroalkenes via Nickel-Catalyzed Allylic Defluorinative Reductive Cross-Coupling. ACS Catal. 2018, 8, 9245-9251. (c) Lin, Z.; Lan, Y.; Wang, C. Reductive Allylic Defluorinative Cross-Coupling Enabled by $\mathrm{Ni} / \mathrm{Ti}$ Cooperative Catalysis. Org. Lett. 2019, 21, 8316-8322. (d) Lin, Z.; Lan, Y.; Wang, C. Synthesis of gem-Difluoroalkenes via Nickel-Catalyzed Reductive C-F and C-O Bond Cleavage. ACS Catal. 2019, 9, 775-780. (e) Lu, X.; Wang, X.-X.; Gong, T.-J.; Pi, J.-J.; He, S.-J.; Fu, Y. Nickel-Catalyzed Allylic Defluorinative Alkylation of Trifluoromethyl Alkenes with Reductive Decarboxylation of RedoxActive Esters. Chem. Sci. 2019, 10, 809-814. (f) Zhao, X.; Li, C.; Wang, B.; Cao, S. Copper-Catalyzed Synthesis of gem-Difluoroallylboronates from $\alpha$-Trifluoromethyl Alkenes and $\mathrm{B}_{2} \operatorname{pin}_{2}$. Tetrahedron Lett. 2019, 60, 129-132.

(37) (a) Lang, S. B.; Wiles, R. J.; Kelly, C. B.; Molander, G. A. Photoredox Generation of Carbon-Centered Radicals Enables the Construction of 1,1-Difluoroalkene Carbonyl Mimics. Angew. Chem., Int. Ed. 2017, 56, 15073-15077. (b) Xiao, T.; Li, L.; Zhou, L. Synthesis of Functionalized gem-Difluoroalkenes via a Photocatalytic Decarboxylative/Defluorinative Reaction. J. Org. Chem. 2016, 81, 7908-7916. (c) Chen, H.; Anand, D.; Zhou, L. Photoredox Defluorinative Alkylation of 1-Trifluoromethyl Alkenes and 1,3Butadienes with 1,4-Dihydropyridines as Alkylation Reagents. Asian J. Org. Chem. 2019, 8, 661-664. (d) Phelan, J. P.; Lang, S. B.; Sim, J.; Berritt, S.; Peat, A. J.; Billings, K.; Fan, L.; Molander, G. A. Open-Air Alkylation Reactions in Photoredox-Catalyzed DNA-Encoded Library Synthesis. J. Am. Chem. Soc. 2019, 141, 3723-3732. (e) Wiles, R. J.; Phelan, J. P.; Molander, G. A. Metal-free Defluorinative Arylation of Trifluoromethyl Alkenes via Photoredox Catalysis. Chem. Commun. 2019, 55, 7599-7602. (f) Wu, L.-H.; Cheng, J.-K.; Shen, L.; Shen, Z.L.; Loh, T.-P. Visible Light-Mediated Trifluoromethylation of Fluorinated Alkenes via C-F Bond Cleavage. Adv. Synth. Catal. 2018, 360, 3894-3899. (g) Guo, Y.-Q.; Wang, R.; Song, H.; Liu, Y.; Wang, Q. Visible-Light-Induced Deoxygenation/Defluorination Protocol for Synthesis of $\gamma, \gamma$-Difluoroallylic Ketones. Org. Lett. 2020, 22, 709-713.

(38) Uneyama, K.; Katagiri, T.; Amii, H. $\alpha$-Trifluoromethylated Carbanion Synthons. Acc. Chem. Res. 2008, 41, 817-829.

(39) (a) Zhang, C. Application of Langlois' Reagent in Trifluoromethylation Reactions. Adv. Synth. Catal. 2014, 356, 2895-2906. (b) Koike, T.; Akita, M. New Horizons of Photocatalytic Fluoromethylative Difunctionalization of Alkenes. Chem. 2018, 4, 409-437. (c) Alonso, C.; Martínez de Marigorta, E.; Rubiales, G.; Palacios, F. Carbon Trifluoromethylation Reactions of Hydrocarbon Derivatives and Heteroarenes. Chem. Rev. 2015, 115, 1847-1935. (d) Pan, X.; Xia, H.; Wu, J. Recent Advances in Photoinduced Trifluoromethylation and Difluoroalkylation. Org. Chem. Front. 2016, 3, 1163-1185.
(40) In this case, the protonated byproduct was isolated in $24 \%$ yield.

(41) Donabauer, K.; Maity, M.; Berger, A. L.; Huff, G. S.; Crespi, S.; König, B. Photocatalytic Carbanion Generation - Benzylation of Aliphatic Aldehydes to Secondary Alcohols. Chem. Sci. 2019, 10, 5162-5166.

(42) Lowry, M. S.; Goldsmith, J. I.; Slinker, J. D.; Rohl, R.; Pascal, R. A.; Malliaras, G. G.; Bernhard, S. Single-Layer Electroluminescent Devices and Photoinduced Hydrogen Production from An Ionic Iridium (III) Complex. Chem. Mater. 2005, 17, 5712-5719.

(43) (a) Jiang, M.; Li, H.; Yang, H.; Fu, H. Room-Temperature Arylation of Thiols: Breakthrough with Aryl Chlorides. Angew. Chem., Int. Ed. 2017, 56, 874-879. (b) Liu, D.; Ma, H.-X.; Fang, P.; Mei, T.S. Nickel-Catalyzed Thiolation of Aryl Halides and Heteroaryl Halides through Electrochemistry. Angew. Chem., Int. Ed. 2019, 58, 5033-5037.

(44) Xu, P.; Li, W.; Xie, J.; Zhu, C. Exploration of C-H Transformations of Aldehyde Hydrazones: Radical Strategies and Beyond. Acc. Chem. Res. 2018, 51, 484-495. 